\title{
Editorial
}

\section{Nuclear Fuel Reprocessing Technologies and Commercialization}

\author{
Michael F. Simpson \\ Department of Metallurgical Engineering, University of Utah, Salt Lake City, UT, USA \\ Correspondence should be addressed to Michael F. Simpson; michael.simpson@utah.edu \\ Received 22 August 2013; Accepted 22 August 2013 \\ Copyright (c) 2013 Michael F. Simpson. This is an open access article distributed under the Creative Commons Attribution License, \\ which permits unrestricted use, distribution, and reproduction in any medium, provided the original work is properly cited.
}

The subject of this special issue of Science and Technology of Nuclear Installations is considered to be a very timely subject given the current expansion of nuclear energy and the lack of broadly accepted long-term spent fuel management solutions. Resource sustainability is also a concern with most projections predicting less than 100 years of easily and therefore economically recoverable natural uranium resources left at current consumption rates. The United States and several other countries are spending tens of millions of dollars each year on nuclear fuel reprocessing research, but there must be a connection to the needs of the existing nuclear power industry. Research at the US national level currently targets the development of a fully sustainable nuclear power infrastructure that maximizes resource utilization and power production, while managing wastes effectively and minimizing the risk of proliferation. Many technologies have been developed over the past half-century that address these areas, but a well-coordinated program is needed to assure efficient implementation. The utilities desire a process for managing the actinides in their used fuel stockpiles that does not impact their reactor operations. The Fukushima disaster has caused many nations to reevaluate how they currently manage spent nuclear fuel and their plans for future disposal or recycling. Given the existing infrastructure, it is unquestionably cheaper today to mine and enrich uranium. However, will significant expansion of nuclear power strain resources sufficiently to swing the pendulum toward reprocessing? Will the externalities associated with waste disposal soon have an impact on the cost of newly mined fuel and provide economic benefit to industry using reprocessed fuel? Can we manage the risks associated with nuclear fuel reprocessing to reap the benefits of better resource utilization and long-term waste management? These were some of the questions posed to the world's experts on nuclear fuel reprocessing. The papers that were received addressed both aqueous and pyrochemical reprocessing. And they touched upon three important issues that span all technologies-safeguards, economics, and waste management.

The concept of safeguards is undoubtedly a critical issue for the commercialization of nuclear fuel reprocessing, given the potential for separating or concentrating plutonium in the various processes being considered. Three papers are included that address this issue. M. Suzuki and N. Terao from Japan Atomic Energy Agency evaluated aqueous solution monitoring as a safeguards approach to reprocessing. This is an example of how the concept of safeguards by design can be implemented. The authors demonstrated via proliferation risk analysis the potential utility of this approach but noted that it is not immune to operator falsification. Meanwhile, T. Burr and colleagues from Los Alamos National Laboratory also addressed the issue of safeguards and utilization of process monitoring data. They studied alarm threshold estimation with the aim of minimizing the frequency of false alarms for nuclear material diversion. It was recommended to use process simulations to determine the optimal alarm thresholds. Not surprisingly, the more accurate the process simulation, the smaller the data sample size needed to establish appropriate alarm threshold levels. They also presented the application of process monitoring for near real-time accounting. In that case, they determined that data could be used as it is received rather than waiting for long periods of time to calculate cumulative material unaccounted for (MUF).

Economics was addressed by two of the papers-one by M. U. Mas et al. of Universidad Pontificia Comillas on a general comparison of open to closed fuel cycles and one by S. K. Kim et al. from Korea Atomic Energy Research Institute 
on the sodium fast reactor and the associated pyrochemical reprocessing scheme. Interestingly, $\mathrm{M}$. U. Mas et al. made the argument that the closed fuel cycle's costs can be reasonably estimated while much uncertainty remains with the longterm cost of the open fuel cycle. This argument largely hinges on the potential downside of unknown costs tied to long-term nuclear waste management. They also make the point that costs associated with deep geologic disposal likely increase as time passes and no action is taken, while reprocessing costs likely decrease due to the benefits of continued research and development. As time passes, it is argued that reprocessing becomes an increasingly compelling choice. A similar conclusion was drawn by S. K. Kim et al. who showed that the estimated cost for fabricating sodium fast reactor metallic fuel via a fuel cycle facility is currently quite close to the economic break-even point. Potential error in this analysis is acknowledged based on the lack of commercial sodium fast reactor fuel cycle facilities in the world, while also noting that costs are expected to come down as the technology is scaled up and widely implemented.

Finally, M. F. Simpson also contributed to this special issue in a paper on the projected salt waste from using the pyroprocessing approach to nuclear fuel reprocessing. This waste salt comes from the oxide reduction and/or electrorefining unit operations and is largely comprised of $\mathrm{LiCl}$ or $\mathrm{LiCl}-\mathrm{KCl}$. A significant range of salt waste generation rates was calculated based on various assumptions. The potential benefit from applying separation processes to recycle the $\mathrm{LiCl}$ or $\mathrm{LiCl}-\mathrm{KCl}$ is quantified. And the trade-offs between disposing of the salt in a waste form and directly without treatment are given. While pyroprocessing has often been touted as having waste management benefits, it is clear that challenges exist in this area and process options must be carefully considered for overall fuel cycle optimization.

Collectively, it appears that nuclear fuel reprocessing does have great potential for commercialization. But there is much work to be done to derive optimal solutions to problems such as safeguards, economics, and waste management. It is, thus, expected that nations will wisely continue to invest in this very important research area.

Michael F. Simpson 


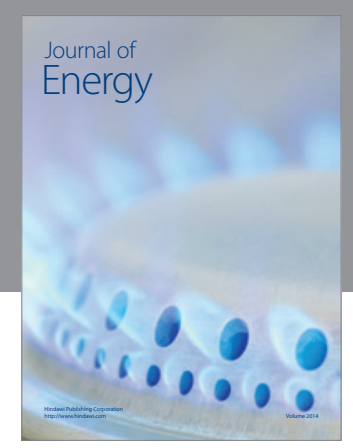

Journal of

Industrial Engineering
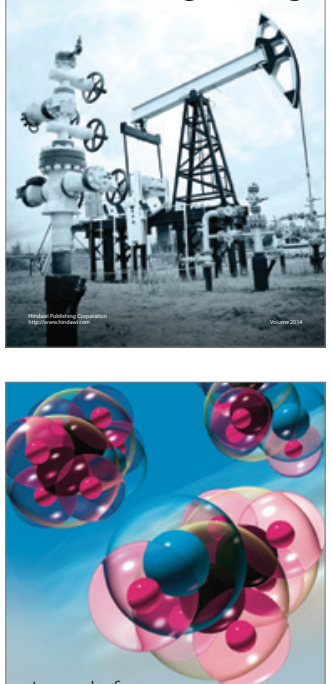

Fuels
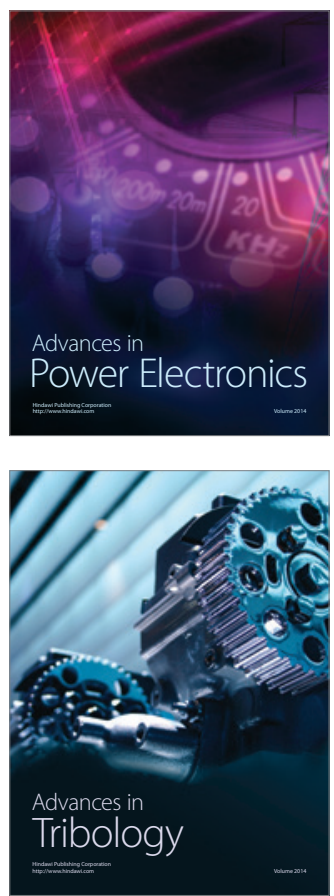

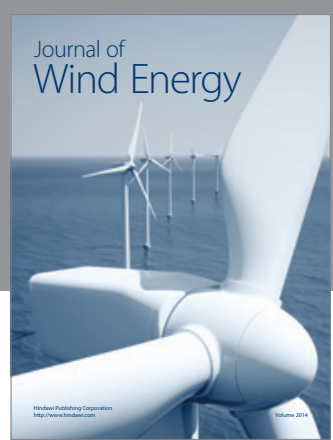

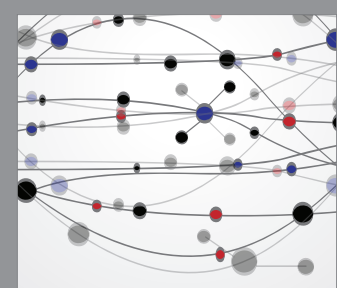

The Scientific World Journal

Submit your manuscripts at http://www.hindawi.com

Journal of

Structures
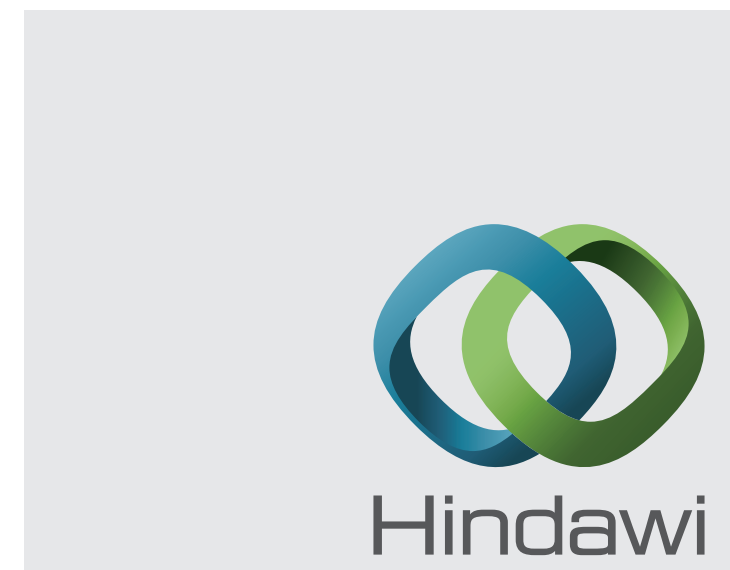

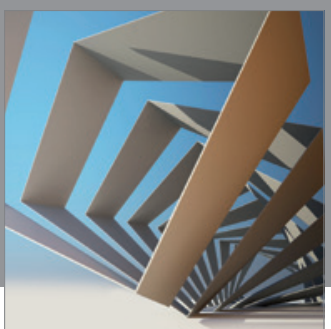

Rotating

Machinery
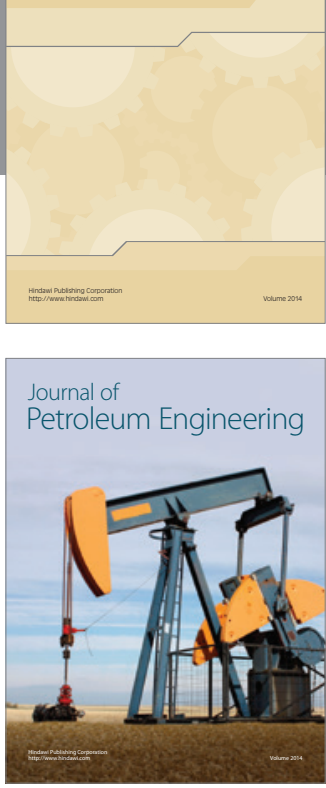

Journal of

Solar Energy
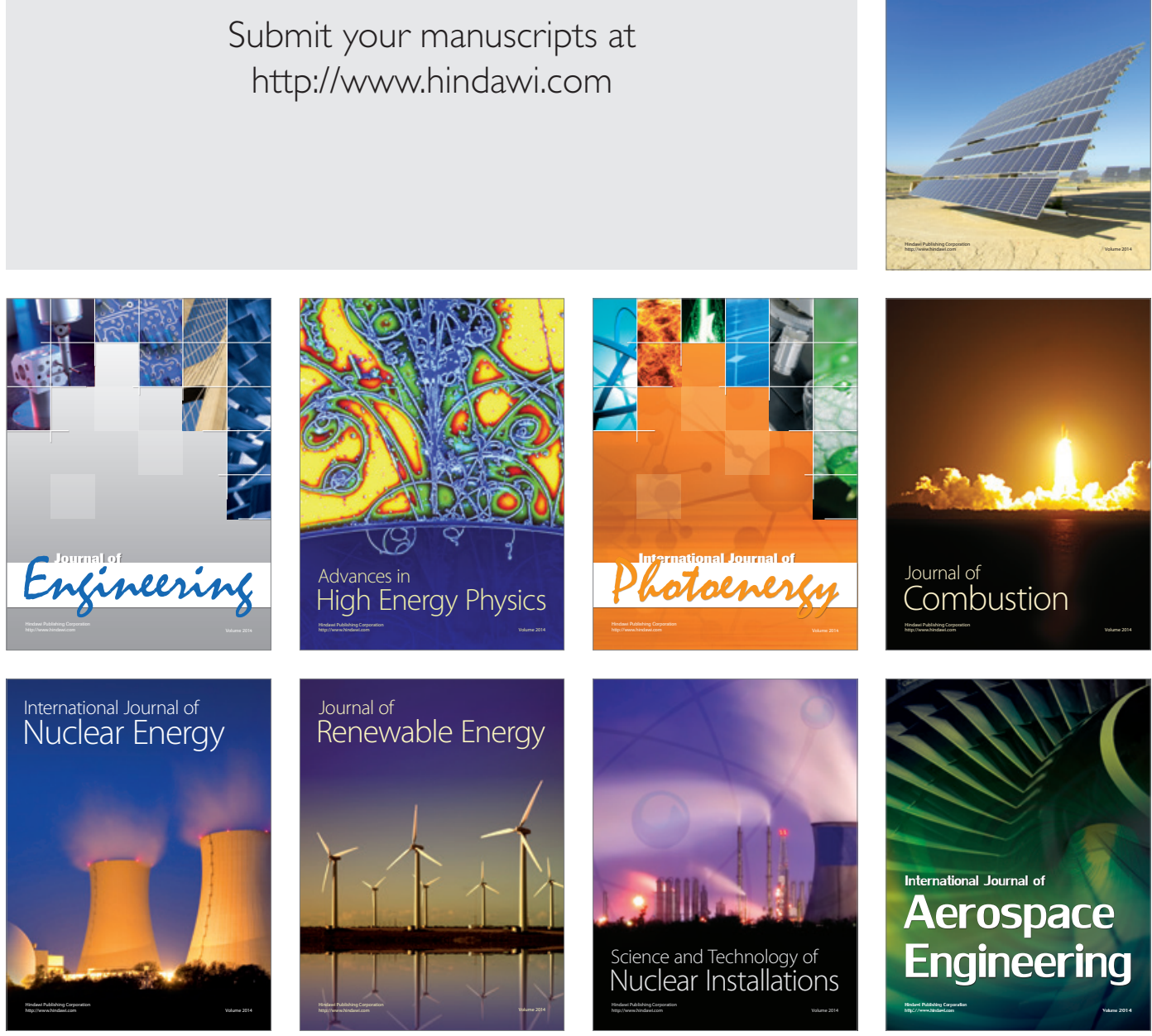